\title{
Nass use and associated factors among outpatients in northern Afghanistan: A cross-sectional study in Andkhoy City
}

\author{
Mohammad Hasssan Hamrah ${ }^{1,2}$, Mohammad Shoaib Hamrah ${ }^{3}$, Mohammad Hussain Hamrah $^{1,4}$, Toba Dahi5, Akbar \\ Fotouhi', Junichi Sakamoto7, Mohammad Hashem Hamrah ${ }^{7}$
}

\begin{abstract}
INTRODUCTION Tobacco use is one of the main risk factors for a number of chronic diseases, including cancer, lung diseases, and cardiovascular diseases. Nass is a form of smokeless tobacco. It is used most commonly in Central Asia, Afghanistan, Pakistan, and Iran, and is significantly associated with oral and oesophageal cancers. The aim of this study was to determine the prevalence of nass use and its associated factors among patients attending an outpatient clinic in Afghanistan.

METHODS This cross-sectional study was performed in an outpatient clinic in Andkhoy, Afghanistan, from April to July 2017. The study included 387 consecutive patients. The data collection tool was a questionnaire, which includes three main items: demographic, physical, and biomedical measurements. We performed binary logistic regression to identify independent risk factors of nass use.

RESULTS The study included 387 participants, of whom 225 were males (58.1\%) and 162 were females $(41.9 \%)$. The overall prevalence of nass use was $48.8 \%$ (95\% CI: 43.8-53.9\%), with the Turkmen ethnic group having significantly higher prevalence than other ethnic groups $(58.2 \%, 95 \%$ CI: $50.8-65.3 \%$ vs $41.8 \%, 95 \%$ CI: $34.7-49.2 \%)$. The mean and standard deviation of the age was 63.9 (17.2) years for nass users and 42.3 (17.4) years for non-users.

Based on a multivariate analysis, family history of nass use (OR=31.4, 95\% CI: 12.5-78.5), illiteracy ( $\mathrm{OR}=8.9,95 \% \mathrm{CI}: 2.5-31.2)$, rural residence $(\mathrm{OR}=2.0,95 \%$ CI: 1.0-3.9), and unemployment ( $\mathrm{OR}=5.4,95 \%$ CI: 1.4-21.4) were associated with nass use.

CONCLUSIONS Our results indicate that about half of the participants were nass users, and nass use associated factors were family history of nass use, illiteracy, unemployment, and rural residency in outpatient clinics in Afghanistan. More surveillance data are needed on a national level to inform the development of strategies for the prevention and control of nass consumption in Afghanistan.
\end{abstract}

https://doi.org/10.18332/tid/93574

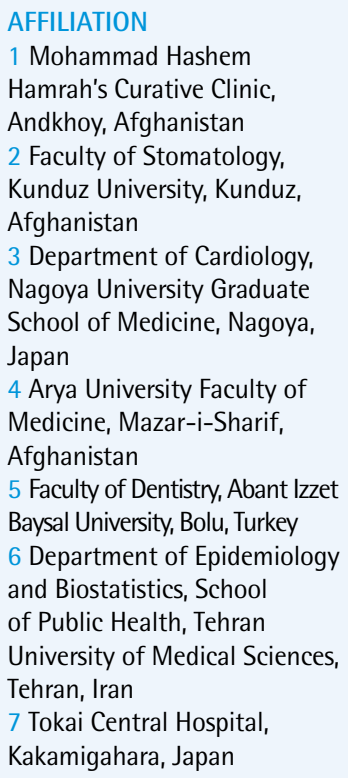

CORRESPONDENCE TO Mohammad Shoaib Hamrah. Department of Cardiology, Nagoya University Graduate School of Medicine,

Nagoya, Japan. E-mail: mshoaibhamrah@gmail.com

KEYWORDS

associated factors,

Afghanistan, nass use

Received: 17 December 2017 Revised: 30 May 2018

Accepted: 18 July 2018

\section{INTRODUCTION}

Tobacco use is a major risk factor for many chronic diseases including cancer, lung diseases, and cardiovascular diseases ${ }^{1}$. Annually, tobacco use accounts for about 7 million deaths globally. More people die from tobacco use than from HIV/AIDS, malaria and tuberculosis combined ${ }^{2}$. The current trend shows that the number of deaths attributed to tobacco use will rise to more than 8 million annually by $2030^{3}$. Tobacco can either be smoked or consumed in a smokeless form. Smokeless tobacco (SLT) causes oral, head and throat cancer ${ }^{4,5}$.

The prevalence of smoking has been decreasing in most developed countries but increasing in 
low-and middle-income countries where $80 \%$ of smokers live ${ }^{6}$. However, the use of SLT products in many developed and developing countries has not changed ${ }^{7}$. Nearly $20 \%$ of the world's tobacco is consumed in smokeless form. Its consumption is common amongst people in South Asia ${ }^{8}$. The use of SLT is more common amongst people in Asia, Africa and the Middle East than in western countries 9 .

SLT is one of the major risk factors associated with the high prevalence of oral, head and throat cancer in South Asia ${ }^{10}$. There are different types of SLT products in different parts of the world, such as traditional chewing tobacco and snuff, and new products such as compressed tobacco lozenges, tobacco chewing gum, and dissolvable strips ${ }^{11}$. SLT is called nass in Afghanistan, Iran, Pakistan, and in central Asian Republics. Nass is a mixture of tobacco leaves, lime, cotton oil or sesame oil, and ash ${ }^{12}$. Nass is used by placing it in the oral cavity under the tongue or between the oral mucosa and gingival cavity ${ }^{13}$. A previous study conducted in Pakistan reported that the chemical constituents of nass are linked to cancer. Nass is attributed to oral and esophageal cancers ${ }^{14}$. The risk of developing oral cancer is more than 20 times higher in nass users compared to nonusers $^{15}$.

Due to three decades of conflict, there is little information available about the prevalence of nass use in Afghanistan. According to a recent study conducted in Kabul regarding the burden of hypertension, the prevalence of nass use among adults was reported to be $9.8 \%{ }^{16}$. Verifying data on the burden and factors associated with nass use among the Afghan population might provide a better understanding of the pattern of nass use and help in developing interventions and health policies. Therefore, the aim of this study was to determine the prevalence of and factors associated with nass use among patients attending an outpatient clinic in Afghanistan.

\section{METHODS}

\section{Study design}

This cross-sectional study was conducted in an outpatient clinic in Andkhoy, Afghanistan, from April 2017 to July 2017. The northern part of Afghanistan was identified as a suitable place to study this smokeless tobacco product and its associated factors.
In all, 387 consecutive patients were included in the study. In brief, the case subjects were patients $18-80$ years old who were physically able and willing to participate. The exclusion criteria were patients being very ill or over 80 years old. The study was approved by the scientific review committee of Balkh Regional Hospital.

\section{Measurements}

The patients were evaluated during their clinic visit using the WHO STEPwise questionnaire ${ }^{17}$, which includes three main items: demographic, physical, and biochemical measurements. The questionnaire was modified by expanding on some optional questions in order to make them more relevant to the specific type of tobacco use. Nass use was included in the survey protocol. The questionnaire was translated into the Dari language and tested to check the effectiveness of the research process (the results of the pilot study was not included in the analysis).

Trained doctors collected relevant data on the nass use, demographic, and clinical characteristics of participants using the WHO STEPwise questionnaire. Demographic variables included items on age, sex, educational level (illiterate, primary/private education, secondary education, university/other higher education), marital status (single, married, other), ethnicity (Turkmen or other ethnic groups), occupation (employed, unemployed, housewife, farmer), place of residence (rural or urban), and participants' age groups $(\leqslant 24,25-44$ and $\geqslant 45$ years). The study protocol included assessment of data obtained from each patient. The second part of the questionnaire included data on other factors outlined below.

\section{Body mass index (BMI)}

The procedure for calculating the BMI (weight in kilograms divided by height in meters squared) of participants was as follows: height was measured without shoes to the nearest $0.5 \mathrm{~cm}$. Before measuring weight, we asked the patients to remove heavy items from their pockets and remove heavy items of clothing or apparel (i.e. big jackets, shoes). We used an electronic scale with accuracy to the nearest $100 \mathrm{~g}$. The procedure for measuring weight was as follows: the scale was zeroed before the patient stepped onto it, then asked to look straight ahead and 
stay still on the scale, while the researcher waited for the measurement to stabilize before recording it.

The definition of BMI as a measure of obesity varies across studies, and even within the same country. For this study, overweight was defined ${ }^{18}$ by a BMI of 25 to $<30 \mathrm{~kg} / \mathrm{m}^{2}$ and obese by a BMI $\geqslant 30$ $\mathrm{kg} / \mathrm{m}^{2}$.

\section{Hypertension}

Patients were considered to be suffering from hypertension if they were on antihypertensive medication at the time of participating in the study or the past medical history/record documented raised blood pressure (BP) at multiple occasions or the $\mathrm{BP}$ was recorded higher than average $(>140 / 90$ $\mathrm{mmHg}$ ) on multiple separate occasions ${ }^{19}$.

\section{Laboratory tests}

Fasting blood sugar (FBS), total cholesterol, and triglycerides (TG) were measured by using HP-CHEM250Y autoanalyzer. The cut-offs for abnormal levels were: $T G \geqslant 150 \mathrm{mg} / \mathrm{dL}$, total cholesterol $\geqslant 200 \mathrm{mg} / \mathrm{dL}$; while individuals with a random blood sugar of $\geqslant 200 \mathrm{mg} / \mathrm{dL}$ were later confirmed by FBS, with an FBS $\geqslant 126 \mathrm{mg} / \mathrm{dL}$ considered diabetic ${ }^{20}$.

\section{Nass use}

In this section, some questions were asked about nass use (status, frequency, duration). The information about nass use was obtained through face-to-face interviews. Respondents were classified as follows: current nass users reported using nass once or more times within the past 30 days, past nass users were defined as lifetime regular users not currently using nass. Non-users were defined as having not consumed nass in the their lifetime $\mathrm{e}^{21,22}$.

\section{Statistical analysis}

All analyses were performed with the SPSS 24.0 software package (SPSS, Armonk, NY: IBM Corp). Nass use rates are shown with $95 \%$ confidence intervals (CIs) for current nass users. Continuous and categorical data were compared using the t-test and $\chi^{2}$-test, respectively. Binary logistic regression was performed to identify the factors associated with nass consumption. A $p<0.05$ was considered significant.

\section{RESULTS}

Participant characteristics are summarized in Table 1. A total of 387 patients were included in this study, of these, 189 were nass users and 198 were nonusers. The mean age and standard deviation was 63.9 (17.2) years for nass users and 42.3 (17.4) years for non-users. The male-to-female ratio among patients was 1.4 , while that in a previous study was $0.6^{23}$. The overall prevalence of nass use was $48.8 \%$ (95\% CI:

\section{Table 1. Distribution of factors associated with nass consumption among the participants}

\begin{tabular}{|c|c|c|c|}
\hline & $\begin{array}{l}\text { Users } \\
\mathrm{N}=189\end{array}$ & $\begin{array}{l}\text { Non-users } \\
\text { V } 198\end{array}$ & $p$ \\
\hline Age, mean (SD), years & $63.9(17.2)$ & $42.3(17.4)$ & $<0.001$ \\
\hline Sex, n (\%) & & & 0.663 \\
\hline Males & $112(59.2)$ & $113(57.0)$ & \\
\hline Females & $77(40.7)$ & $85(42.9)$ & \\
\hline Level of education, n (\%) & & & $<0.001$ \\
\hline Illiterate & $169(89.4)$ & $90(45.4)$ & \\
\hline Primary/private education & $8(4.2)$ & $28(14.1)$ & \\
\hline Secondary & $4(2.1)$ & $33(16.6)$ & \\
\hline High school or more & $8(4.2)$ & $47(23.7)$ & \\
\hline Marital Status, n (\%) & & & 0.282 \\
\hline Single & $34(18.0)$ & $25(12.6)$ & \\
\hline Married & $146(77.2)$ & $160(80.8)$ & \\
\hline Others & $9(4.8)$ & $13(6.5)$ & \\
\hline Ethnicity, n (\%) & & & $<0.001$ \\
\hline Turkmen & $110(58.2)$ & $56(28.3)$ & \\
\hline Other ethnic groups & $79(41.8)$ & $142(71.7)$ & \\
\hline Occupation, n (\%) & & & 0.001 \\
\hline Employed & $47(24.9)$ & 79 (39.8) & \\
\hline Unemployed & $14(7.4)$ & $24(12.1)$ & \\
\hline Housewife & $51(27.0)$ & $44(22.2)$ & \\
\hline Farmers & $77(40.7)$ & $51(25.7)$ & \\
\hline Place of residence, $n(\%)$ & & & $<0.001$ \\
\hline Rural & $132(69.8)$ & $62(31.3)$ & \\
\hline Urban & $57(30.2)$ & $136(68.7)$ & \\
\hline $\begin{array}{l}\text { History of family nass use, } \\
n(\%)\end{array}$ & $98(51.8)$ & $10(5.0)$ & $<0.001$ \\
\hline \multicolumn{4}{|l|}{$\begin{array}{l}\text { Measurements and tests, } \\
\text { mean (SD) }\end{array}$} \\
\hline $\begin{array}{l}\text { Systolic blood pressure } \\
(\mathrm{mmHg})\end{array}$ & $129.3 \pm 22.0$ & $136.0 \pm 23.9$ & 0.004 \\
\hline $\begin{array}{l}\text { Diastolic blood pressure } \\
(\mathrm{mmHg})\end{array}$ & $65.4 \pm 12.7$ & $89.1 \pm 16.3$ & 0.013 \\
\hline Body mass index $\left(\mathrm{kg} / \mathrm{m}^{2}\right)$ & $23.4 \pm 3.1$ & $23.5 \pm 3.9$ & 0.835 \\
\hline Fasting blood sugar (mg/dL) & $108.2 \pm 42.8$ & $104.4 \pm 25.7$ & 0.290 \\
\hline Total cholesterol (mg/dL) & $174.1 \pm 30.4$ & $173.1 \pm 32.7$ & 0.744 \\
\hline Triglyceride (mg/dL) & $159.5 \pm 38.9$ & $160.3 \pm 38.4$ & 0.836 \\
\hline
\end{tabular}


43.8-53.9\%), with the Turkmen ethnic group having significantly higher prevalence than other ethnic groups (58.2\%, 95\% CI: $50.8-65.3 \%$ vs $41.8 \%$, 95\% CI: $34.7-49.2 \%)$. The majority of nass users (89.4\%) were illiterate. The nass use was significantly higher among unemployed patients $(27.0 \%$ vs $22.2 \%$; $\mathrm{p}=0.001)$. Rural residence was associated with nass use $(69.8 \%$ vs $32.8 \%$; $<<0.001)$. The employment rates were significantly lower among nass users than non-users $(24.8 \%$ vs $39.8 \%$; $=0.001)$. Rural residence was associated with nass use $(69.8 \%$ vs $28.8 \% ; \mathrm{p}<0.001)$. There was a significant difference in the mean (SD) systolic pressure between patients who were nass users compared with non-users [129 (22) vs 136 (24); p=0.004]. The mean diastolic blood pressure was significantly lower among nass users than non-users [65.4 (12.7) vs 89.1 (16.3); $\mathrm{p}=0.013]$.

Table 2. Odd ratio of risk factors for nass consumption among study participants

\begin{tabular}{|c|c|c|c|c|c|}
\hline Variables & N & $\begin{array}{l}\text { Vass } \\
\text { users }\end{array}$ & ; OR & $\left(95^{\circ} \% \mathrm{CI}\right)$ & p \\
\hline \multicolumn{6}{|l|}{$\begin{array}{l}\text { Family member } \\
\text { using nass }\end{array}$} \\
\hline No & 279 & 91 & 1.0 & & \\
\hline Yes & 108 & 98 & 31.4 & $(12.5-78.5)$ & $<0.001$ \\
\hline \multicolumn{6}{|l|}{ Education } \\
\hline $\begin{array}{l}\text { High school or } \\
\text { more }\end{array}$ & 55 & 8 & 1.0 & & \\
\hline Secondary & 37 & 4 & 5.1 & $(1.7-15.4)$ & 0.004 \\
\hline $\begin{array}{l}\text { Primary/private } \\
\text { education }\end{array}$ & 36 & 8 & 5.0 & $(1.4-17.9)$ & 0.014 \\
\hline Illiterate & 259 & 169 & 8.9 & $(2.5-31.2)$ & 0.001 \\
\hline \multicolumn{6}{|l|}{ Occupation } \\
\hline Employed & 126 & 47 & 1.0 & & \\
\hline Housewife & 95 & 51 & 1.0 & $(0.5-2.8)$ & 0.017 \\
\hline Farmers & 128 & 77 & 0.4 & $(0.2-0.8)$ & 0.927 \\
\hline Unemployed & 38 & 14 & 5.4 & $(1.4-21.4)$ & 0.017 \\
\hline \multicolumn{6}{|l|}{ Residence place } \\
\hline Urban residents & 193 & 62 & 1.0 & & \\
\hline Rural residents & 194 & 132 & 2.0 & $(1.0-3.9)$ & 0.044 \\
\hline \multicolumn{6}{|l|}{ Age, years } \\
\hline$\leq 24$ & 36 & 6 & 1.0 & & \\
\hline $25-44$ & 102 & 22 & 0.3 & $(0.1-1.7)$ & 0.175 \\
\hline$\geq 45$ & 249 & 161 & 0.1 & $(0-0.4)$ & 0.003 \\
\hline \multicolumn{6}{|l|}{ Ethnicity } \\
\hline Other ethnic groups & 221 & 79 & 1.0 & & \\
\hline $\begin{array}{l}\text { Turkmen ethnic } \\
\text { group }\end{array}$ & 166 & 110 & 1.8 & $(0.9-3.3)$ & 0.074 \\
\hline
\end{tabular}

Table 2 shows the results of a binary logistic regression model determining the relation between nass use and family history of nass use, level of education, rural residency, occupation status, ethnicity, and age.

Nass use was considered a dependent variable. Patients with family history of nass use were 31.4 times more likely to use nass (95\% CI: 12.5-78.5). The odds of nass use were 8.9 higher among individuals with no education compared to those with high school or tertiary education (95\% CI: 2.578.5 ). The odds of nass use among rural residents were 2.0 times higher compared to urban residents (95\% CI: 1.0-3.9), while the odds of the unemployed using nass were 5.4 times higher (95\% CI: 1.4-21.4) than for those who were employed.

\section{DISCUSSION}

To our knowledge, this is the first study to investigate the prevalence of nass use and its predictors among the Afghan population. SLT use has traditionally been part of the society and culture of the people of South and Southeast Asia ${ }^{24}$. Previous studies from Karachi showed that nass use played a strong role in the etiology of oral cancer in Pakistan ${ }^{25,26}$. The findings of our study showed that about half of the patients were using nass. Nass use was associated with the factors: family history of nass use, illiteracy, unemployment, and rural residency.

The present study showed that there was a high level of nass consumption among adult patients who visited an outpatient clinic in Andkhoy, Afghanistan. This is in line with a previous study conducted with adult patients who visited family medicine clinics in Karachi, Pakistan ${ }^{27}$. The reasons for the high rates of nass consumption might be that nass use is more acceptable among people in this region, it is inexpensive, easily available, and easier to use than cigarettes. There is also no law prohibiting nass sales, the sale of nass is not monitored in Afghanistan, and some smokers may also supplement their tobacco dependency habit by nass use. While there are antitobacco campaigns in Afghanistan, these mainly target cigarette smokers and messages against nass are often absent. It is also possible that due to the increasing taxation on cigarettes by the government of Afghanistan, the high cigarette prices might be a reason for some smokers to consider nass use as 
an alternative to smoking. Another reason for the high rate of nass use could be industry marketing: in recent years, nass is being sold in more attractive and colorful sachets and has also been made more flavorsome.

Afghanistan has ratified the WHO Framework Convention on Tobacco Control (FCTC) and on 2 Feb 2015 the law on tobacco control was enacted. The national law, which is supported by Islamic Shari'ah Law, includes banning the production, promotion, and use of cigarettes, water-pipe, hookah, snuff, and other tobacco products. It aims to help tobacco users to quit tobacco use, warn about the dangers of tobacco, and raise taxes on tobacco. However, the laws are not implemented exactly as they should be in Afghanistan ${ }^{28,29}$.

In our study, the use of nass was higher among the Turkmen ethnic group compared to other ethnic groups. One possible explanation might be that use of nass has been socially and culturally acceptable for Turkmen people for generations. For this reason, Turkmen nass consumers believe that nass use is part of their tradition.

Use of nass was higher among those participants who had at least one family member that used nass. A similar finding has been reported in another study among adult patients who visited family practice clinics in Pakistan ${ }^{30}$. It is believed that the role of family members in the initiation of nass use is more significant than that of society, peers, or other factors ${ }^{31}$. Moreover, when the habit is initiated before 18 years of age, the first lesson of smoking and tobacco consumption is often acquired at home from family members ${ }^{32}$. The most likely reason might be easy access to nass at home.

In our study, nass consumption is inversely related to the level of education. Similar findings have been reported from a previous study ${ }^{33}$. This might be due to the lack of awareness about the harmful effects of SLT and also that the death rate and overall risk taking behavior tends to be higher among the less educated ${ }^{34}$. It is likely that lack of awareness about the harmful effects of nass will lead the less educated in Afghanistan to situations predisposing them to nass use. It is also possible that the anti-tobacco educational campaigns initiated by the Afghan Ministry of Public Heath are limited in their effectiveness.
In our study, nass use was higher among unemployed people. This association with poverty has been seen in a previous study ${ }^{35}$. It is believed that tobacco may be used as a coping mechanism associated with the reduction of stress ${ }^{36}$.

Our finding is that nass use is prevalent among rural residents, which is similar to results of previous surveys in Bangladesh ${ }^{37}$. This could be due to lack of awareness among rural residents. Also, for socioeconomically disadvantaged Afghan people in rural areas, nass is locally manufactured, inexpensive, and easily available.

\section{Limitations}

This study has some limitations. The sample size consisted of a single-center data source. The single-center nature of this study may affect its generalizability to the entire population of Afghanistan. Furthermore, the patients were selected on a convenience basis, which could have led to a potential source of bias. In addition, the information about nass use was obtained through face-to-face interviews administered through a structured questionnaire. Due to social reasons associated with tobacco and nass use, people may under-report their nass use. Therefore, selfreported nass use among participants is only reliable when tested by a serum cotinine level as indicator of nicotine metabolism among people who use both smoking and smokeless tobacco ${ }^{38}$. In addition, we determined the prevalence of nass use in a clinical setting. However, conducting this study among the wider population would be more effective in truly representing the prevalence of nass use. Furthermore, this study is based on a non-probability sample, and therefore cannot be generalized beyond the study population. Also, we did not perform a statistical test like Cronbach's alpha for the questionnaire and there was only face validity for the questionnaire.

Finally, the cross-sectional nature of the study makes it difficult to interpret the correlation between nass use and its associated factors.

On the other hand, our data can be valuable for the accumulation of additional data regarding nass use, as our study was the first on prevalence and risk factors of nass use among adult patients attending an outpatient clinic in Afghanistan. 


\section{CONCLUSIONS}

This study showed that about half of the participants who were adult patients attending an outpatient clinic in Afghanistan were nass users. Nass use was more common among the Turkmen ethnic group. Factors associated with nass use were family history of nass use, illiteracy, unemployment, and rural residency. Our findings greatly reinforce the need to develop strategies for the control of nass consumption in Afghanistan.

\section{REFERENCES}

1. Jayes L, Haslam PL, Gratziou CG, et al. SmokeHaz: Systematic reviews and meta-analyses of the effects of smoking on respiratory health. Chest. 2016;150:164179. doi:10.1016/j.chest.2016.03.060

2. World Health Organization. WHO report on the global tobacco epidemic, 2017. Monitoring tobacco use and prevention policies. Executive summary. http://apps. who.int/iris/bitstream/handle/10665/258503/WHONMH-PND-17.4-eng.pdf?sequence=1. Published, 2017. Accessed December 17, 2017.

3. World Health Organization. WHO report on the global tobacco epidemic, 2011: warning about the dangers of tobacco. Most. 2011;152:1-152. http://www.who. int/tobacco/global_report/2011/exec_summary/en/. Accessed December 17, 2017.

4. Balaram P, Sridhar H, Rajkumar T, et al. Oral cancer in Southern India: the influence of smoking, drinking, paan-chewing and oral hygiene. Int J Cancer. 2002;98(3):440-445. doi:10.1002/ijc.10200

5. Johnson N. Tobacco use and oral cancer: a global perspective. J Dent Educ. 2001;65(4):328-339.

6. Reitsma MB, Fullman $\mathrm{N}, \mathrm{Ng} \mathrm{M}$, et al. Smoking prevalence and attributable disease burden in 195 countries and territories, 1990-2015: a systematic analysis from the Global Burden of Disease Study 2015. Lancet. 2017;389(10082):1885-1906. doi:10.1016/S0140-6736(17)30819-X

7. Agaku IT, Ayo-Yusuf OA, Vardavas CI, Connolly G. Predictors and patterns of cigarette and smokeless tobacco use among adolescents in 32 countries, 2007-2011. J Adolesc Heal. 2014;54(1):47-53. doi:10.1016/j.jadohealth.2013.07.037

8. Khan A, Huque R, Shah SK, et al. Smokeless Tobacco Control Policies in South Asia: A Gap Analysis and Recommendations. Nicotine Tob Res. 2014;16(6):890894. doi:10.1093/ntr/ntu020

9. Gupta R, Gupta N, Khedar RS. Smokeless tobacco and cardiovascular disease in low and middle income countries. Indian Heart J. 2013;65(4):369-377. doi:10.1016/j.ihj.2013.06.005

10. Khan Z, Tönnies J, Müller S. Smokeless tobacco and oral cancer in South Asia: A systematic review with meta-analysis. J Cancer Epidemiol. 2014. doi:10.1155/2014/394696

11. Gupta PC, Ray CS. Smokeless tobacco and health in India and South Asia. Respirology. 2003;8(4):419-431. doi:10.1046/j.1440-1843.2003.00507.x

12. Roland M, Asma S, Backinger C. Smokeless tobacco fact sheets. Third Int Conf Smokeless Tob. 2002:1-24.

13. Sajid F, Bano S. Effects of smokeless dipping tobacco (naswar) consumption on antioxidant enzymes and lipid profile in its users. Pak J Pharm Sci. 2015;28(5):18291833.

14. Zakiullah, Saeed M, Muhammad N, et al. Assessment of potential toxicity of a smokeless tobacco product (naswar) available on the Pakistani market. Tob Control. 2012;21(4):396-401. doi:10.1136/tc.2010.042630

15. Khan Z, Dreger S, Shah SMH, et al. Oral cancer via the bargain bin: the risk of oral cancer associated with a smokeless tobacco product (Naswar). PLoS One. 2017;12(7). doi:10.1371/journal.pone.0180445

16. Saeed KMI. Burden of hypertension in the capital of Afghanistan: a cross-sectional study in Kabul City, 2015. Int J Hypertens. 2017. doi:10.1155/2017/3483872

17. World Health Organization. STEPS instrument for NCD risk factors: WHO STEP wise approach to surveillance of noncommunicable diseases (STEPS). http://www. who.int/ncd_surveillance. Published, 2015. Accessed December 17, 2017.

18. Musaiger AO. Overweight and obesity in Eastern Mediterranean region: prevalence and possible causes. J Obes. 2011. doi:10.1155/2011/407237

19. Carretero OA, Oparil S. Essential Hypertension. Circulation. 2000;101(3):329-335. doi:10.1161/01.cir.101.3.329

20. National Cholesterol Education Program (NCEP) Expert Panel on Detection Evaluation and Treatment of High Blood Cholesterol in Adults (Adult Treatment Panel III). Third report of the National Cholesterol Education Program (NCEP) Expert Panel on Detection, Evaluation, and Treatment of High Blood Cholesterol in Adults (Adult Treatment Panel III) final report. Circulation. 2002;106(25):3143-3421.

21. Howard-Pitney B, Winkleby MA. Chewing tobacco: who uses and who quits? Findings from NHANES III, 1988-1994. Am J Public Health. 2002;92(2):250-256. doi:10.2105/AJPH.92.2.250

22. Nelson DE, Mowery P, Tomar S, Marcus S, Giovino G, Zhao L. Trends in smokeless tobacco use among adults and adolescents in the United States. Am J Public Health. 2006;96(5):897-905. doi: 10.2105/AJPH.2004.061580

23. Hamrah MS, Hamrah MH, Ishii H, et al. Association between Helicobacter pylori infection and cardiovascular risk factors among patients in the northern part of Afghanistan: a cross-sectional study in Andkhoy city. Asian Pac J Cancer Prev. 2018;19(4):1035-1039. doi:10.22034/APJCP.2018.19.4.1035 
24. Dhanani R, Jafferani A, Bhulani N, Azam SI, Khuwaja AK. Predictors of oral tobacco use among young adult patients visiting family medicine clinics in Karachi, Pakistan. Asian Pac J Cancer Prev. 2011;12(1):43-47.

25. Jafarey NA, Mahmood Z, Zaidi SH. Habits and dietary pattern of cases of carcinoma of the oral cavity and oropharynx. J Pak Med Assoc. 1977;27(6):340-343.

26. Merchant A, Husain SSM, Hosain M, et al. Paan without tobacco: an independent risk factor for oral cancer. Int J Cancer. 2000;86(1):128-131. doi:10.1002/(SICI)10970215(20000401)86:1<128::AID-IJC20>3.0.CO;2-M

27. Ali NS, Khuwaja AK, Ali T, Hameed R. Smokeless tobacco use among adult patients who visited family practice clinics in Karachi, Pakistan. J Oral Pathol Med. 2009;38(5):416-421. doi:10.1111/j.1600-0714.2009.00754.x

28. WHO report on the global tobacco epidemic, 2017. Country profile: Afghanistan. http://www.who.int/ tobacco/surveillance/policy/country_profile/afg.pdf. Published, 2017. Accessed December 17, 2017.

29. Official Gazette. http://untobaccocontrol.org/impldb/ wp-content/uploads/reports/afghanistan_2016_ annex1_tobacco_control_act_2015_EN.pdf. Accessed December 17, 2017.

30. Ali NS1, Khuwaja AK, Ali T, Hameed R. Smokeless tobacco use among adult patients who visited family practice clinics in Karachi, Pakistan. J Oral Pathol Med. 2009 May;38(5):416-21.

31. Rozi S, Akhtar S. Prevalence and predictors of smokeless tobacco use among high-school males in Karachi, Pakistan. East Mediterr Heal J. 2007;13(4):916-924.

32. Dwivedi S, Aggarwal A, Singh N, Aggarwal S, Sharma $\mathrm{V}$. Role of family milieu in tobacco addiction: a study in a tertiary-care institution in India. J Heal Popul Nutr. 2013;31(1):130-132. doi:10.1111/josh.12381

33. Gupta PC. Survey of sociodemographic characteristics of tobacco use among 99,598 individuals in Bombay, India using handheld computers. Tob Control. 1996. doi:10.1136/tc.5.2.114

34. Khawaja MR, Mazahir S, Majeed A, et al. Chewing of betel, areca and tobacco: perceptions and knowledge regarding their role in head and neck cancers in an urban squatter settlement in Pakistan. Asian Pac J Cancer Prev. 2006;7(1):95-100.

35. Rooban T, Elizabeth J, Umadevi KR, Ranganathan K. Sociodemographic correlates of male chewable smokeless tobacco users in India: a preliminary report of analysis of National Family Health Survey, 20052006. Indian J Cancer. 2010;47(Suppl)1:91-100. doi:10.4103/0019-509X.63865

36. Costa PT, McCrae RR. Stress, smoking motives, and psychological well-being: The illusory benefits of smoking. Adv Behav Res Ther. 1981;3(4). doi:10.1016/0146-6402(81)90001-1

37. Global Adult Tobacco Survey Bangladesh Report 2009.
World Health Organization; 2009. http://www.searo. who.int/bangladesh/publications/gats_2009/en/. Accessed December 17, 2017.

38. Wagenknecht LE, Burke GL, Perkins LL, Haley NJ, Friedman GD. Misclassification of smoking status in the CARDIA study: a comparison of self-report with serum cotinine levels. Am J Public Health. 1992;82(1):33-36. doi:10.2105/AJPH.82.1.33
ACKNOWLEDGEMENTS

This project was supported in part, by the non-profit organization Epidemiological and Clinical Research Information Network (ECRIN). MSH is supported by the Fellowship of Takeda Science Foundation. We would like to thank Dr Mirwais Rabi for his cooperation with this study.

CONFLICTS OF INTEREST Authors have completed and submitted the ICMJE Form for Disclosure of Potential Conflicts of Interest and none was reported.

FUNDING

There was no source of funding for this research.

PROVENANCE AND PEER REVIEW

Not commissioned; externally peer reviewed. 